\title{
¿La combinación de clones posee alguna ventaja sobre los sistemas
}

\section{monoclonales? Posibles alternativas silviculturales para las Salicáceas, a}

\section{partir de la revisión de experiencias previas}

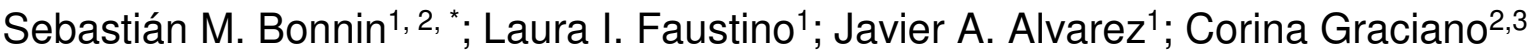 \\ ${ }^{1}$ INTA EEA Delta del Paraná - Río Paraná de las Palmas y Canal Laurentino Comas, 4ta Sección de Islas, \\ (B2804) Campana, Buenos Aires, Argentina; ${ }^{2}$ CONICET - Godoy Cruz 2290 (C1425FQB) CABA, Argentina; ${ }^{3}$ \\ INFIVE (CONICET-Universidad Nacional de La Plata) - Diag. 113 esq. 61, (1900) La Plata, Buenos Aires, \\ Argentina; * bonnin.sebastian@inta.gob.ar; bonninse@gmail.com
}

Bonnin Sebastián M.; Laura I. Faustino; Javier A. Alvarez; Corina Graciano (2020). ¿La combinación de clones posee alguna ventaja sobre los sistemas monoclonales? Posibles alternativas silviculturales para las Salicáceas, a partir de la revisión de experiencias previas. Rev. Fac. Agron. Vol 119 (2): 1-11. https://doi.org/10.24215/16699513e051

Las plantaciones forestales han sido tradicionalmente desarrolladas a partir de material de propagación de origen seminal. Sin embargo, las principales especies plantadas en el mundo se pueden propagar vegetativamente, por lo que las posibilidades para el desarrollo de la silvicultura clonal son crecientes. El cultivo de Salicáceas se realiza con estacas o guías, por lo que las plantaciones son $100 \%$ producidas bajo un esquema de silvicultura clonal. Además, en el Delta del Río Paraná los rodales se instalan como bloques monoclonales y el clon Populus deltoides 'Australiano 129/60' es el más difundido, definiendo una matriz productiva con una estrecha diversidad genética. Esto determina que el sistema sea menos estable y trae aparejado un riesgo productivo ante la aparición de condiciones de estrés biótico o abiótico. En consecuencia, el modelo de silvicultura monoclonal presenta algunas desventajas no solo desde el punto de vista ecológico, sino también productivo. A partir de la posibilidad que brindan las especies forestales, que presentan una gran variabilidad tanto intra como interespecífica, es posible pensar en combinación de clones para aumentar la diversidad genética de las plantaciones. Las plantaciones clonales mixtas pueden favorecer a la disminución de los impactos de las actividades productivas en el ambiente, distribuir espacialmente la demanda de nutrientes del suelo, aumentar la diversidad genética y mejorar los índices de rendimiento del rodal. El objetivo de esta revisión es analizar los antecedentes de silvicultura clonal y plantaciones mixtas como base para la propuesta de alternativas silviculturales para las Salicáceas del Delta del Paraná.

Palabras clave: plantaciones clonales mixtas; silvicultura clonal; competencia intraespecífica; álamos; Delta del Río Paraná

Bonnin Sebastián M.; Laura I. Faustino; Javier A. Alvarez; Corina Graciano (2020). Does clones combination have any advantage in comparison to monoclonal systems? Silvicultural alternatives for Salicaceae, based on the review of previous experiences. Rev. Fac. Agron. Vol 119 (2): 1-11. https://doi.org/10.24215/16699513e051

Forest plantations with different trees and purposes were traditionally developed with seminal seedlings. Actually, the most important planted tree species can be propagated by vegetative methods, so clonal silviculture has high possibilities to grow. Salicaceae plantations are established by cuttings of genetically improved trees. This determines that poplars and willows are produced under clonal silviculture systems. Furthermore, at the region of Paraná River Delta, stands are monoclonal and Populus deltoides 'Australiano 129/60' is the most widespread clone. These forest systems are unstable and there is a productive risk associated to emergence of pests, diseases and extreme climatic conditions. Thus, monoclonal silviculture model has some ecological and productive disadvantages. Reducing genetic diversity could threat the aims of the forest plantations and it is important to consider aspects related to biodiversity when plantations are planned. Clone combination in the same stand can be useful to increase genetic diversity, because tree species are highly diverse at both inter and intraspecific level. Mixed clonal plantations can favor to decrease the impact of productive activities on the environment, spatially distribute soil nutrient demand, increase genetic diversity and enhance stand yields. The aim of this review is to analyze previous experiences in clonal silviculture and plantation in mixed stands as a framework to propose silvicultural alternatives for Salicaceae production in Paraná River Delta, Argentina.

Key words: policlonal plantation; clonal silviculture; intraspecific competition; poplars; Paraná River Delta

https://revistas.unlp.edu.ar/revagro

Recibido: 20/09/2019

Aceptado: 06/06/2020

Disponible on line: $07 / 12 / 2020$

ISSN 0041-8676 - ISSN (on line) 1669-9513, Facultad de Ciencias Agrarias y Forestales, UNLP, Argentina 


\section{DEFINICIÓN DE PLANTACIÓN FORESTAL CLONAL}

La silvicultura comprende las prácticas tendientes a controlar el establecimiento, crecimiento, composición, sanidad y calidad de los bosques naturales o implantados (Ashton \& Kelty, 2018). Las prácticas silvícolas van desde la plantación y los tratamientos culturales durante el período de crecimiento, hasta la cosecha o corta final. El cultivo de árboles de diferentes especies y con diversos fines, ha sido tradicionalmente desarrollado a partir de material de propagación de origen seminal. Sin embargo, las Salicáceas -y otras especies que se propagan vegetativamente de forma natural- se cultivan hace varios siglos mediante el uso de estacas o guías de tallo, por su capacidad de enraizamiento y facilidad para ser multiplicadas de forma agámica. La propagación clonal permite multiplicar los mejores individuos, asegurar la conservación del germoplasma valioso, aumentar la ganancia genética y acortar los ciclos de selección en los programas de mejoramiento genético (Zobel \& Talbert, 1984). Los programas de mejoramiento genético forestal han permitido obtener materiales seleccionados, disminuir las pérdidas durante el establecimiento y mejorar el rendimiento de las plantaciones forestales en general. A partir del desarrollo de nuevas técnicas, comenzó a producirse material de plantación de origen clonal en diferentes partes del mundo. Es decir, nuevas plantas originadas a partir de la multiplicación vegetativa de materiales seleccionados previamente, dando origen a la silvicultura clonal de especies que antes no se podían conducir con este tipo de prácticas. Podríamos entonces, definir a la silvicultura clonal como el cultivo de árboles con fines productivos, de conservación u otros, utilizando como material de plantación plantines, estacas o guías obtenidas por diferentes métodos de propagación agámica. Esto incluye el tradicional enraizamiento de estacas (e.g. Salicáceas), o métodos más complejos como el cultivo in-vitro o ex-vitro. En cualquiera de los casos, la práctica de la silvicultura clonal está ligada a la disponibilidad de un método de propagación vegetativa masivo (Park et al., 1998).

\section{EJEMPLOS DE PLANTACIONES FORESTALES CLONALES}

$\mathrm{Si}$ se tiene en cuenta que las principales especies plantadas en el mundo se pueden propagar vegetativamente mediante diferentes métodos, las posibilidades para el desarrollo de la silvicultura clonal son crecientes. La superficie total mundial de plantaciones forestales ronda los 278 millones de hectáreas (Payn et al., 2015) con la mayor superficie cubierta por los géneros Pinus spp (pinos) y Eucalyptus spp (eucaliptos). Sin embargo, según la región varían las especies más utilizadas para la producción de madera y otros productos forestales: en zonas tropicales y subtropicales, predominan Acacia spp y Eucalyptus spp, junto con Tectona grandis (teca) y Gmelina arbórea. En climas mediterráneos y templados, las especies del género Pinus son las más cultivadas, además de otras especies de los géneros Eucalyptus y Populus. Por otra parte, en las zonas con climas más fríos también se plantan especies de los géneros Pseudotsuga, Abies, Larix y Picea (Prado, 2015). En Argentina, las plantaciones forestales cubren una superficie aproximada de 1.300 .000 ha, con Pinus spp y Eucalyptus spp como los principales géneros de especies plantadas, seguidos por los sauces y álamos (Secretaría de Agroindustria de la Nación, 2018). Potencialmente, toda la superficie de plantaciones forestales podría convertirse en plantaciones clonales, si se desarrolla el método adecuado para cada especie.

En el mundo, además de las Salicáceas, especies de géneros como Picea spp, Larix spp, Gmelina arbórea y Tectona grandis también se cultivan actualmente, en mayor o menor proporción, bajo sistemas clonales. Sin embargo, hay algunas que han sido altamente evaluadas y otras que llevan pocos años de desarrollo en la silvicultura clonal. Por ejemplo, la mitad de las plantaciones de Eucalyptus spp en Brasil, el país del mundo con mayores forestaciones de este género, se realizan bajo sistemas clonales (Wu, 2018). Además, una de las especies más consideradas en el mundo para el establecimiento de plantaciones comerciales es la teca. En Brasil, para el año 2013, existían aproximadamente unas 60.000 ha de teca plantadas principalmente al sur de la región de Mato Grosso, representando la mayor superficie de plantación de esta especie en América Latina. Las técnicas de multiplicación vegetativa han permitido la instalación de algunas plantaciones clonales de teca y ensayos para la evaluación de su productividad (Medeiros et al., 2015). En países como Canadá, donde las coníferas representan las principales especies comerciales, las nuevas técnicas de clonación han resultado una herramienta importante para la multiplicación de los genotipos de interés. La embriogénesis somática permitió el desarrollo de clones seleccionados por una determinada aptitud y testeados a campo. Además, surgieron las Forestaciones Multi-Varietales (MVF) en el este de Canadá, que se basan en instalar plantaciones comerciales mezclando más de un clon (Park et al., 2016). Hasta hace algunas décadas, la silvicultura clonal en Argentina se restringía a los álamos y sauces en el Delta del Paraná, región de Cuyo y Valle del Río Negro en la Patagonia, especies que pueden multiplicarse vegetativamente con facilidad. Actualmente, se producen plantines clonales de eucaliptos y pinos, lo que permitió el avance de las plantaciones clonales en las principales cuencas forestales del país. Algunas empresas e institutos de investigación en Argentina han desarrollado y/o introducido clones de Eucalyptus spp para las forestaciones del litoral. Por ejemplo, el Instituto Nacional de Tecnología Agropecuaria (INTA) tiene registrados 18 clones comerciales en el Registro Nacional de Cultivares (RNC), donde además hay otros 34 clones registrados por empresas del sector. Para el caso de las Salicáceas, existen registrados, 19 clones comerciales de álamos y 12 clones de sauces (Instituto Nacional de Semillas, 2020). La Mesopotamia representa la región forestal por excelencia en el país, y el cultivo de pinos es el que mayor superficie ocupa, concentrado principalmente en las provincias de Corrientes y Misiones. Le siguen los eucaliptos, con preponderancia en la provincia de Entre Ríos. La silvicultura clonal en la Mesopotamia es una práctica relativamente nueva que tuvo grandes avances en los últimos 20 años. Se estima que las plantaciones de origen clonal de eucaliptos representan el $20 \%$ del total 
(Oberschelp, com. pers., 2018), aunque en la actualidad no existen datos oficiales al respecto.

A diferencia de otros géneros, los árboles adultos de Pinus taeda no rebrotan de cepa y son recalcitrantes a la propagación vegetativa, con lo cual la transferencia en forma de clones de los mejores genotipos del programa de mejoramiento, no puede ser trasladada a plantaciones comerciales sin la utilización de la técnica de embriogénesis somática (Gauchat et al., 2015). Debido a esto, aún no se encuentran registrados clones comerciales en el INASE (Instituto Nacional de Semillas) por lo que el cultivo clonal en pinos presenta un nivel de expansión menor que en el caso de los clones de eucaliptos, los cuales se encuentran actualmente disponibles para la comercialización y el acceso por parte de los productores. La región de Sudamérica con mayor superficie forestada con Salicáceas es el Delta del Río Paraná, donde la actividad forestal data de fines del siglo XIX, teniendo una importante historia de producción de madera de álamos y sauces para las industrias del papel, el triturado y el aserrado (Cortizo, 2005a). Como se mencionó anteriormente, el cultivo de Salicáceas se realiza con estacas o guías de materiales mejorados genéticamente, por lo que las plantaciones de la región son 100\% producidas bajo un esquema de silvicultura clonal. Además, los rodales se instalan como bloques monoclonales, no registrándose experiencias de plantaciones policlonales en la región. Por otra parte, a pesar de que se encuentran disponibles en el mercado, un número importante de clones comerciales de álamos, el clon Populus deltoides W. Bartram ex Marshall' Australiano 129/60' es el más difundido, determinando que la matriz productiva a escala de paisaje también sea preponderantemente monoclonal.

\section{VENTAJAS PRODUCTIVAS DE LAS PLANTACIONES CLONALES}

Las plantaciones clonales se han desarrollado con el objetivo de obtener una producción más homogénea y aprovechar al máximo las ganancias genéticas obtenidas en los programas de mejoramiento. La clonación es una herramienta mediante la cual se captura de forma rápida el genotipo de los mejores individuos y como consecuencia, se maximizan los progresos provenientes de la selección en cada ciclo de mejoramiento (Meza et al., 2015). Por otro lado, resulta importante para la propagación de genotipos híbridos, obtenidos a partir del cruzamiento de dos especies de interés. Un ejemplo claro de esta situación son los híbridos de Eucalyptus grandis $\mathrm{x}$ Eucalyptus urophylla que conjugan las características de altas tasas de crecimiento de una especie, con la calidad de fibra para celulosa de la otra. Si bien se pueden obtener plantines de origen seminal, a partir de la propagación vegetativa es posible la multiplicación de los mejores genotipos producto de dichos cruzamientos. Además, permite la selección sitio-específica de clones para la obtención de los genotipos mejor adaptados a cada condición ambiental (Kumar, 2016). En Brasil, por ejemplo, en los programas de mejoramiento genético y de selección clonal hay una tendencia a desarrollar materiales específicos, tanto para una mayor adaptación a las diferentes condiciones edafoclimáticas, como para la búsqueda de diferentes calidades de maderas (Xavier \& da Silva, 2010). La selección de genotipos tolerantes a distintos tipos de estreses abióticos y/o resistentes a enfermedades o plagas frecuentes posibilita la expansión de las plantaciones hacia zonas anteriormente consideradas improductivas, o mejora el rendimiento de las forestaciones en aquellas regiones que presentan condiciones adversas para el crecimiento de los árboles. Es así que la silvicultura clonal se ha vuelto un sistema de producción altamente eficiente y de alto impacto en la rentabilidad del negocio forestal (Carpineti, 2005). No obstante, los plantines clonales tienen un costo elevado respecto de los obtenidos a partir de semillas - a excepción de las Salicáceas - por lo que un análisis económico es necesario para determinar, según la escala de producción, el esquema silvícola más adecuado para el establecimiento forestal. Existen circunstancias en las que emplear plantines de origen seminal es menos arriesgado y más sostenible en lo económico, principalmente si se trata de especies con dificultades para el enraizamiento (Griffin, 2014).

Cabe mencionar que los clones son materiales seleccionados de programas de mejoramiento genético que poseen, por lo general, un alto grado de avance. Por ello, es de esperar que se trate de materiales altamente productivos. Sin embargo, no hay que dejar de considerar la importancia del ambiente en la expresión del genotipo, razón por la cual podemos encontrar materiales genéticos mejorados que no presentan una buena performance en algunos sitios. Ante condiciones de heterogeneidad ambiental, el material seminal puede aportar un nivel de diversidad genética mayor que permita una mejor adaptabilidad de la población al sitio y a la aparición de enfermedades o plagas. La silvicultura clonal ha mejorado la productividad promedio de las plantaciones de eucaliptos en Brasil, desde los 25-30 $\mathrm{m}^{3} \mathrm{ha}^{-1}$ año-1 a los 35$45 \mathrm{~m}^{3} \mathrm{ha}^{-1}$ año-1 en las últimas tres décadas. Estas plantaciones, se suelen realizar estableciendo mosaicos monoclonales (Xavier \& da Silva, 2010). Algunas empresas han obtenido más del $25 \%$ de ganancias genéticas en volumen respecto de plantaciones de origen seminal desarrolladas en los mismo sitios (Rezende et al., 2014). Por otra parte, según datos presentados por Pomera Maderas en Argentina, la empresa ha obtenido una tasa interna de retorno (TIR) del $22 \%$ con la utilización de clones de eucaliptos respecto de una del $16 \%$ obtenida en plantaciones de origen seminal. El incremento medio anual (IMA) ha sido $33 \mathrm{~m}^{3} \mathrm{ha}^{-1}$ año-1 en plantaciones clonales y $27 \mathrm{~m}^{3} \mathrm{ha}^{-1} \mathrm{año}^{-1}$ en las de origen seminal (Vargas, 2015).

Otras especies que ocupan una superficie menor, pero de gran importancia comercial tanto a escala local como global, también fueron evaluadas en distintas partes del mundo. Para el caso de Pinus radiata, a partir de la generación de clones, es posible obtener ganancias genéticas adicionales, duplicando lo que se puede lograr con la silvicultura seminal dentro de una misma generación. Por ejemplo, compañías forestales como Forest Genetics, estiman ganancias de alrededor del 15$20 \%$ en volumen para plantaciones clonales en Nueva Zelanda (Wu, 2018). Además, según Medeiros et al., (2015), en ensayos instalados para evaluar diferentes sistemas de producción de teca, las plantaciones clonales han tenido un mayor rendimiento que las plantaciones 
seminales, además de poseer costos de implantación de alrededor del $3 \%$ inferiores a las plantaciones seminales. Los productos forestales no madereros (PFNM), como el cacao en India, también pueden encontrar ciertas ventajas en la clonación, para obtener altos rendimientos, de forma uniforme y temprana (Apshara, 2017).

\section{DESVENTAJAS DE LAS PLANTACIONES MONOCLONALES}

La conservación de la biodiversidad ha sido una de las mayores preocupaciones de los organismos internacionales en la definición de políticas públicas, respecto al uso de los recursos naturales y el control sobre las actividades productivas que se realizan en los distintos ambientes. Las iniciativas internacionales como el Procesos de Montreal y el Sistema Pan-Europeo han hecho especial énfasis en la conservación de la biodiversidad en los ecosistemas forestales (Castañeda, 2004). A nivel comercial también se han desarrollado mecanismos de certificación con el objetivo de compatibilizar los fines productivos con la conservación y/o mantenimiento de la biodiversidad (Carnus et al., 2006). Los sistemas forestales difieren de otros cultivos, en especial de la agricultura extensiva, por el tiempo durante el cual se encuentran en íntima relación con el ambiente en el que se desarrollan. Las condiciones climáticas adversas, la aparición de nuevas plagas y/o enfermedades, el desarrollo de procesos de erosión de los suelos, entre otras, son algunas de las posibles situaciones a las cuales una plantación forestal debe hacer frente a lo largo de su ciclo productivo. Esto requiere de una gran estabilidad del ecosistema forestal, que algunos autores asocian a la diversidad genética disponible en el mismo. Siguiendo este razonamiento, cuanto más diverso es un sistema, más estable resulta ante situaciones como las mencionadas (Hooper et al., 2005; Jactel et al., 2005; Park et al., 1998). Dicha diversidad genética es un componente importante de la biodiversidad, incluida la variabilidad entre individuos de una misma especie, ya que existe una variación funcional a nivel de genotipo, y no sólo de las especies, que pueden influir en las interacciones competitivas y los procesos de crecimiento en los árboles (Benavides et al., 2018; Boyden et al., 2008). Esta diversidad intraespecífica, en gran parte, controla la aclimatación o resistencia a estreses bióticos o abióticos y tiene un importante rol en el mantenimiento de la sanidad y productividad de los bosques (Carnus et al., 2006; Hamanishi et al., 2015).

El establecimiento de plantaciones forestales monoclonales trae aparejado una disminución drástica en la diversidad genética del sistema, pero se suele justificar su implementación por la capacidad de obtener una producción más homogénea en el tiempo y espacio, además de los rendimientos potencialmente mayores. Sin embargo, a pesar de tratarse de un mismo genotipo, las plantaciones monoclonales también varían internamente en las características de los árboles que las componen (Binkley et al., 2010; Resende et al., 2018), aunque en menor medida que las forestaciones de origen seminal. Esto se debe a la interacción de cada individuo con el ambiente y la relación de competencia por los recursos con sus vecinos (Resende et al., 2016). Un ejemplo claro de las diferencias que pueden mostrar dos individuos genéticamente idénticos, ante variaciones en las condiciones ambientales, es el llamado efecto de bordura. Las plantas que crecen en los márgenes de las plantaciones suelen ser más grandes y desarrollar mayor número de ramas que aquellas que se encuentran en el interior del rodal. Esto se debe a la mayor disponibilidad de luz y puede darse en respuesta a otros estímulos ambientales como la disponibilidad de agua y/o nutrientes, o la presencia/ausencia de estrés micro-ambiental.

La instalación de plantaciones monoclonales, sumado a la utilización de un bajo número de clones en la cuenca forestal, puede generar grandes superficies con una estrecha diversidad genética. Si bien puede tener algunos beneficios desde el punto de vista operacional, el uso de un solo clon elimina la posibilidad de utilizar el mejor genotipo para cada sitio. Además, trae aparejado un riesgo productivo, ya que la aparición de una enfermedad, plaga - situación climática extrema podría tener efectos devastadores en toda la región (Larjavaara, 2008; Park et al., 2016). Por otra parte, estudios de percepción muestran que, en términos generales, las plantaciones heterogéneas tienen una mayor aceptación por parte de la comunidad que los sistemas homogéneos (Ribe, 1989; Edwards et al., 2012; Felton et al., 2016; Filyushkina et al., 2017; Almeida et al., 2018). Las variables analizadas incluyen la composición de los rodales, las prácticas silviculturales como podas y raleos, la edad y tamaño de los árboles, la utilización de especies exóticas y el diseño de los sistemas forestales a escala de paisaje, entre otras (Gundersen \& Frivold, 2008).

En consecuencia, el modelo de silvicultura monoclonal presenta algunas desventajas no solo desde el punto de vista ecológico, sino también productivo, ya que de acuerdo con la hipótesis de partición de recursos (Ammer, 2019; Grossiord, 2019), los vecinos que son similares en sus necesidades o su ecofisiología tienen una mayor superposición de su nicho ecológico y menos oportunidades para la complementariedad en el uso de los recursos (Hector \& Loreau, 2001), lo que conduce a una competencia más intensa (Boyden et al., 2008). Esto puede determinar una menor disponibilidad de recursos y una disminución en los rendimientos de rodales monoclonales, en comparación con rodales policlonales (Elferjani et al., 2014).

El meta-análisis realizado por Jactel et al. (2005) sostiene la teoría generalizada de que los sistemas con monocultivos son más propensos al ataque de plagas que los sistemas más diversos. Esto se debe a que cuanto más diverso es el rodal, más difícil es para el insecto encontrar el hospedante y mayor es su riesgo de mortalidad durante el proceso de búsqueda (Jones, 2001). Por otra parte, enfermedades de origen fúngico que pueden tener una alta especificidad con sus hospedantes, pueden experimentar un brote generalizado si resultan compatibles con los clones que ocupan la mayor parte de la superficie cultivada. Esta es una problemática que ha tenido ejemplos evidentes a lo largo de la historia en el Delta del Río Paraná, donde en varias oportunidades hubo clones que se dejaron de plantar debido al ataque generalizado de patógenos y/o plagas. Por ejemplo, las primeras plantaciones de álamo en el Delta del Paraná se realizaron con Populus deltoides subesp. angulata cv. carolinensis, que sufrió severos ataques de roya (Melampsora spp) y fue abandonado por los productores. Años más tarde, los clones "Catfish 2" y "Catfish 5", los 
álamos más plantados en la década de 1980, debieron ser reemplazados por ataques generalizados de roya que devastaron las plantaciones de la región (Cortizo, 2005b). En este sentido, las plantaciones de Salicáceas en Argentina han servido de ejemplo para evidenciar la problemática asociada al establecimiento de grandes superficies con una baja diversidad genética.

\section{INTERACCIONES EN RODALES CLONALES MIXTOS}

Si tenemos en cuenta que los niveles de acción pueden ir desde el rodal hasta una cuenca forestal completa, las estrategias para mantener una matriz productiva genéticamente diversa pueden incluir tanto el establecimiento de rodales monoclonales con la utilización de varios clones -formando mosaicos en un paisaje heterogéneo-, hasta la combinación de clones dentro de un mismo rodal (Figura 1). Para disminuir el riesgo implícito en grandes extensiones monoclonales, el uso de unos 30 a 50 clones de orígenes genéticos no emparentados constituye una buena estrategia para sostener operacionalmente un programa de plantación comercial de unas 5000 ha año-1 (Carpineti, 2005). Park et al. (1998) determinan que la utilización de 15 a 25 clones es un número suficiente en términos de protección, manteniendo aún los beneficios de la silvicultura clonal. Estos conceptos podrían aplicarse independientemente de la especie en cuestión, aunque estas estrategias se enmarcan en esquemas que combinan rodales monoclonales. A partir de la necesidad de compatibilizar los objetivos productivos con el mantenimiento de la biodiversidad, podría considerarse como alternativa silvicultural el establecimiento de plantaciones clonales mixtas. Estos sistemas mixtos tendrían además una ventaja respecto de la combinación de distintas especies: combinar un mismo material de plantación hace que operativamente sean menos complejas y que muchas de las tareas silvícolas se mantengan sin grandes modificaciones. Sin embargo, hay que considerar que al momento de la plantación, el material debe estar correctamente identificado y esto puede demandar mayor tiempo que en la plantación de rodales monoclonales.
En plantaciones mixtas se distinguen 3 tipos de interacciones benéficas principales: 1- partición de los recursos; 2- facilitación y 3- efecto de selección (Forrester \& Bauhus, 2016; Grossiord, 2019). La partición de los recursos, refiere a las diferencias en características funcionales, que disminuyen la competencia por los mismos (e.g., diferente profundidad de raíces, consumo de agua o arquitectura de copa). La facilitación hace alusión a los mecanismos que ocurren cuando un individuo tiene un efecto positivo sobre sus vecinos (e.g., mediante la capacidad de redistribuir agua desde la napa hacia horizontes superficiales más secos, la simbiosis con fijadores de nitrógeno, o la capacidad de oxigenar suelos anegados mediante la formación de aerénquimas). Finalmente, el efecto de selección se relaciona con los procesos selectivos que determinan la dominancia de individuos con características particulares (e.g., frente a estreses bióticos y abióticos, dominarán los genotipos más tolerantes).

A partir de la amplia diversidad genética que poseen las especies forestales en general, y las Salicáceas en particular, es posible adaptar algunos de estos conceptos para el análisis de las interacciones entre diferentes clones de una misma especie. En la región del Delta del Río Paraná, los clones más utilizados en las plantaciones forestales pertenecen en su mayoría, a la especie Populus deltoides (Borodowski, 2014; Cortizo et al., 2014). Sin embargo, estos clones presentan variabilidad en características como la duración del área foliar (DAF) y las fechas de brotación y abscisión foliar (Bonnin et al., 2018). Por lo tanto, si se combinan clones de manera que los árboles de brotación temprana estén rodeados por individuos de clones de brotación tardía, los primeros tendrán mayor disponibilidad de luz al inicio de la estación de crecimiento. Esto permitirá que aumente su capacidad de fijar carbono. Algo similar ocurre con cambios en la fecha de abscisión otoñal: los de abscisión tardía tendrán mayor disponibilidad de luz una vez que sus vecinos estén defoliados.

Por otra parte, existen diferencias entre clones de álamo en la concentración de nutrientes en hojas y raíces durante la estación de crecimiento, como así también durante el invierno, relacionado con diferente retranslocación de nutrientes (Faustino et al., 2016). a)

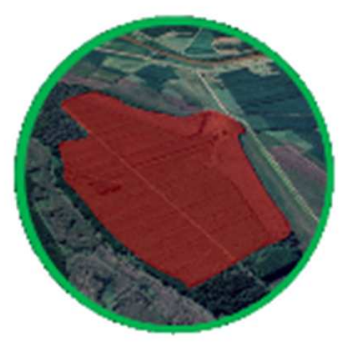

MENos

DIVERSO b)

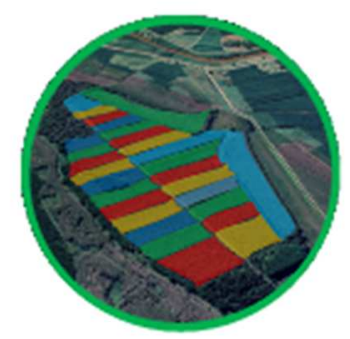

c)

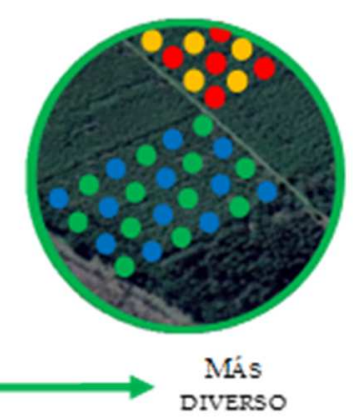

Figura 1. Niveles para la diversificación de una cuenca forestal: a) predio monoclonal; b) predio policlonal con rodales monoclonales; c) combinación de clones en un rodal. 
En Pinus taeda, también existe variación en la acumulación de nitrógeno en hojas entre familias selectas por su elevado crecimiento (Faustino et al., 2013). Complementar clones o familias con diferente uso y distribución de nutrientes puede reducir la tasa de extracción de nutrientes del suelo e influir en la composición de la hojarasca. Esto tendrá un impacto en la mineralización de la materia orgánica y circulación de nutrientes.

Asimismo, en álamo también existen diferencias clonales en las respuestas a condiciones de estrés por sequía, inundación y salinidad (Guarnaschelli et al., 2011; Faustino, 2013; Alvarez et al., 2015; Elferjani et al., 2016; Faustino et al., 2016; Russo et al., 2018). La tolerancia a la sequía se debe a cambios en la partición de materia seca (e.g., en la mayor proporción de raíces y tallos que abastecen de agua a las hojas) y en respuestas en el corto plazo, como el cierre estomático que evita pérdidas excesivas de agua, cuando baja la disponibilidad de agua en el suelo o la demanda evapotranspirativa de la atmósfera es elevada (Faustino, 2013; Alvarez, 2018). Si se combinan clones, que posean raíces con diferente profundidad de exploración del suelo, en situaciones de sequía la disponibilidad de agua para cada clon será mayor que si cada individuo estuviera rodeado por vecinos del mismo clon que extraen agua del mismo sector del perfil. Asimismo, si un clon cierra los estomas frente a un estrés hídrico leve, consumirá menos agua, de manera que quedará más agua disponible en el suelo para el clon que no haya cerrado los estomas. En cuanto a la tolerancia a la inundación, un aspecto crucial es abastecer de oxígeno a las raíces. Hay clones de álamo que tienen la capacidad de generar raíces adventicias superficiales, lenticelas hipertrofiadas y aerénquimas (Luquez et al., 2012; Rodriguez, 2014) que además de permitir la supervivencia de ese individuo, oxigenan el suelo, de manera que individuos que no pueden generar aerénquimas serán beneficiados.

Este tipo de diferencias en la morfología, fisiología y fenología de los árboles, es determinante para el desarrollo de mecanismos de complementariedad (Forrester \& Bauhus, 2016) (Figuras 2 y 3). Es posible entonces, pensar que esto se traduzca en diferencias en la captación de luz, la dinámica hídrica de los árboles y el ciclado de nutrientes en el sistema. Esto habilita la posibilidad de encontrar las mejores interacciones genotipo-ambiente (y entre genotipos), y de esta forma establecer plantaciones forestales que sean altamente productivas, pero con mejores indicadores de biodiversidad.

Además, frente a un estrés abiótico, que altera los procesos fisiológicos, las plantas resistentes a dicho estrés, pueden responder con mecanismos que van desde la tolerancia a la evitación (Lambers et al., 2008). Las plantas tolerantes mantendrán los procesos fisiológicos relativamente estables aún en la condición de estrés, mientras que las evitadoras utilizarán recursos para salir de dicha condición de estrés (Joly \& Crawford, 1982; Lopez \& Kursar, 1999). Dado que a nivel de clones pueden existir genotipos no resistentes, tolerantes y evitadores a cada estrés abiótico (Guo et al., 2011; Peng et al., 2013; Russo et al., 2018), la plantación clonal mixta puede ser una alternativa superadora, que lleve a rendimientos mayores que las plantaciones monoclonales (Moghaddam, 2014). Por lo tanto, es importante conocer los requerimientos de recursos (i.e., luz, agua y nutrientes) y la tolerancia a los estreses bióticos y abióticos de los posibles materiales genéticos a ser mezclados, de manera de seleccionar clones que se complementen en sus requerimientos y mecanismos de aclimatación.

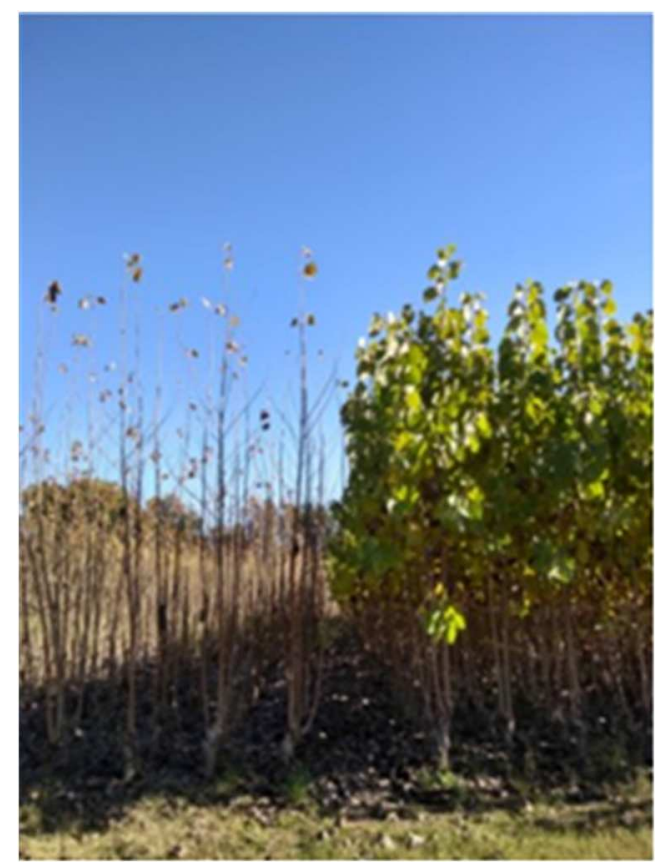

Figura 2. Diferencias en las fechas de abscisión foliar entre dos clones de P. deltoides del Programa de Mejoramiento Genético de INTA EEA Delta del Paraná (Argentina). Izq: $P$. deltoides 'Paycarabí INTA'; der: $P$. deltoides 'Nandi INTA'.

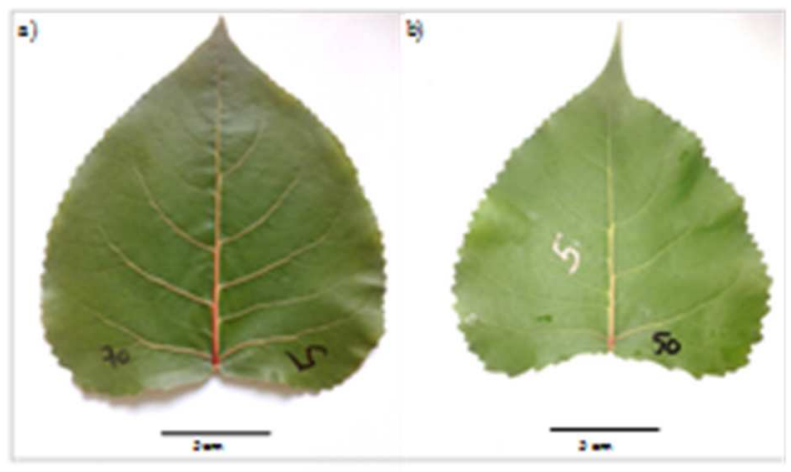

Figura 3. Diferencias morfológicas (e.g., tamaño, forma y color) de las hojas entre dos clones de $P$. deltoides del Programa de Mejoramiento Genético de INTA EEA Delta del Paraná (Argentina). a) $P$. deltoides 'Hovyú INTA'; b) $P$. deltoides 'Guayraca INTA'.

En cuanto a los estreses bióticos, a partir de la posibilidad que brindan las especies forestales que presentan una gran variabilidad entre especies pero también, entre 
individuos de una misma especie (Boyden et al., 2008), es posible pensar en la combinación de clones con fundamentos similares a los que sostienen quienes proponen las plantaciones de especies mixtas. Los beneficios de este tipo de forestaciones, consisten principalmente en la propagación del riesgo entre los árboles que tienen diferente susceptibilidad a factores de estrés (Bauhus et al., 2017). Por ejemplo, la existencia de clones resistentes puede significar una barrera a la expansión de las enfermedades y disminuir el riesgo para aquellos individuos susceptibles (McCracken \& Dawson, 1997). McCracken \& Dawson (1998) determinaron el retraso en la aparición de roya y un menor nivel de la enfermedad hacia el final de la temporada de crecimiento, en parcelas con mezcla de clones de sauces, cuando se comparaban con los mismos clones en sus respectivas parcelas monoclonales.

La alta productividad a nivel de rodal es una característica importante en la producción de madera, y está asociada al secuestro de carbono y otros nutrientes. Un concepto clave para el diseño de rodales mixtos altamente productivos es la necesidad de combinar individuos que difieran en características como la tolerancia al sombreado, la tasa de crecimiento en altura, la densidad de área foliar, la fenología foliar y la profundidad de raíces (Kelty, 2006). La alta variabilidad existente entre clones de álamo, permite pensar en estrategias de uso de los recursos diferentes (Faustino et al., 2016; Guarnaschelli et

\section{al., 2011) (Figura 4).}

Si los individuos difieren sustancialmente en las características mencionadas, podrían capturar recursos del sitio más eficientemente, resultando en una mayor producción total de biomasa en el rodal que la que ocurriría en una plantación monoclonal (Kelty, 2006; Ammer, 2019). La estrategia de combinación de clones a nivel de rodal, resulta interesante ya que pueden existir diferencias en la plasticidad fenotípica de algunos clones en respuesta al ambiente (Guet et al., 2015; Elferjani et al., 2016; Faustino et al., 2016; Alvarez, 2018). Incluso en rodales homogéneos, existe variabilidad dada por la heterogeneidad ambiental y la relación entre el crecimiento individual y el crecimiento de los árboles vecinos (Resende et al., 2018). Además, la eficiencia en el uso de los recursos puede diferir entre individuos en una plantación y determinar cambios en el crecimiento de los árboles (Fernández \& Gyenge, 2009; Binkley et al., 2010). Estas interacciones competitivas dentro del rodal, pueden derivar en mayores rendimientos debido a una menor competencia intraespecífica (Kelty, 2006; Boyden et al., 2008; Elferjani et al., 2014). Los mayores rendimientos en rodales clonales mixtos se podrían lograr por mayor crecimiento individual (i.e., menor competencia con igual densidad de plantación) o con mayor densidad de plantación, pero manteniendo la intensidad de competencia del rodal monoclonal (Resende et al., 2018).

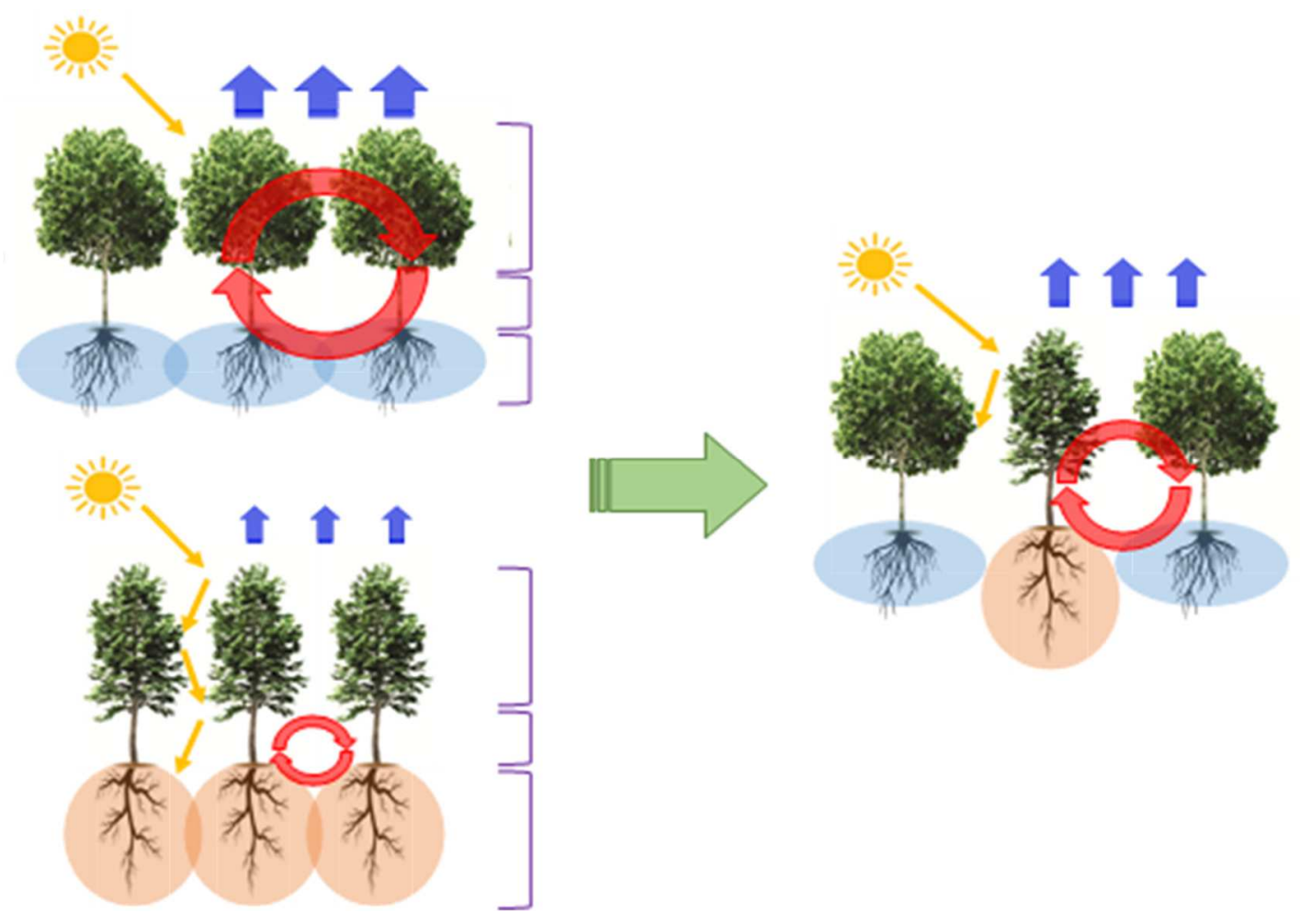

Figura 4. Esquema de potenciales diferencias entre clones de álamo en la captación y uso de los recursos en rodales monoclonales (izquierda) y rodales mixtos (derecha). Las flechas azules simbolizan la evapotranspiración del sistema; las flechas amarillas, la radiación incidente; los ciclos rojos representan la tasa de retranslocación y el ciclado de nutrientes; los corchetes violetas destacan la asignación de biomasa a cada compartimento del árbol; y las burbujas en las raíces simbolizan el área de exploración del suelo y nicho de cada clon. 
En este sentido, la partición de recursos mencionada anteriormente, es una de las teorías ecológicas que puede adaptarse a sistemas con combinación de clones. Por ejemplo, en plantaciones clonales mixtas de álamo en Canadá, se observaron mayores valores de biomasa aérea, diferencias en la distribución de raíces y en la disponibilidad de nutrientes del suelo, comparándolas con las parcelas monoclonales. Los clones utilizados mejoraron su performance cuando se mezclaban, pero el diferencial positivo fue aún mayor para los clones menos productivos. Además, las diferencias se acentuaron en los sitios más pobres en nutrientes (Elferjani et al., 2014). Sin embargo, el tipo y nivel de respuesta a la mezcla de clones dependerá de los genotipos específicos que se involucren y del sitio de plantación (Foster et al., 1998; Mina et al., 2018). La respuesta a la mezcla de más de un clon en el crecimiento depende de las interacciones entre los clones utilizados, y de la densidad y la edad de la plantación ya que el grado de interacción entre los individuos aumenta con la edad de los rodales (Benbrahim et al., 2000).

La propuesta del rodal clonal mixto tiene algunas de las ventajas que se les asignan a las plantaciones mixtas y podría ser fácilmente adoptada por los productores, porque no requeriría de grandes cambios en las prácticas silviculturales habituales. Esto es importante porque la dificultad en el manejo de plantaciones mixtas con especies muy diferentes, puede limitar operacionalmente el establecimiento de este tipo de plantaciones (Nichols et al., 2006; Prado, 2015).

Para optimizar los beneficios de la mezcla de clones, es importante tener información previa de los materiales genéticos que nos permita elegir la mejor opción. Más allá de que el aumento de la diversidad tiene un valor en sí mismo, desde el punto de vista productivo se requiere llevar adelante una "mezcla inteligente". De lo contrario, podrían surgir algunos inconvenientes debido a la combinación de genotipos con diferencias extremas. Por ejemplo, si las tasas de crecimiento no son parejas, uno de los clones podría establecerse como dominante en el rodal y perjudicar el crecimiento de los otros. Además, podrían diferir en el momento óptimo para la cosecha. Respecto de las tareas silvícolas, si los clones difieren demasiado en la forma de la copa o el número de ramas, podrían requerir distinto tratamiento o número de podas. No obstante, resulta importante mencionar que para la región del Delta del Paraná, los materiales genéticos disponibles provienen de programas de mejoramiento y han sido seleccionados con fines productivos. Esto determina que, a pesar de las diferencias morfofisiológicas que presentan, los clones comerciales tengan niveles de rendimiento relativamente parejos. Además, han sido evaluados respecto de la calidad de la madera y aceptados por la industria local como aptos (Cerrillo, 2014; Cortizo et al., 2017).

\section{CONSIDERACIONES FINALES}

Las plantaciones forestales necesitan de un programa de manejo sustentable que garantice el éxito de la producción, pero reduzca al mínimo posible los impactos negativos en el ambiente y el riesgo de pérdidas productivas de las mismas. Una diversidad genética reducida puede atentar contra los objetivos por los cuales se realizó una plantación, por lo que resulta de gran importancia incluir aspectos relacionados con la biodiversidad en la realización de un plan de manejo forestal. Según el ámbito de toma de decisiones, variará el nivel en el cual se pueda actuar. El productor forestal podrá decidir el esquema de manejo de sus plantaciones a escala de rodal y/o predio, y las autoridades correspondientes deberán ser las encargadas de llevar adelante políticas que fomenten la diversificación a escala de cuenca o paisaje.

En resumen, la discusión sobre la combinación de clones suele darse en dos aspectos: por un lado, los posibles beneficios que trae aparejado un aumento de la variabilidad genética desde el punto de vista ecológico en general, y en cuanto a la protección forestal en particular. Por otra parte, las posibilidades de obtener un aumento en el rendimiento debido a una menor competencia intraespecífica y una complementariedad en el uso de los recursos. Dependerá de los clones en cuestión y del contexto en el que se desarrollen, el tipo y nivel de interacciones que se desencadenen en el rodal.

Para facilitar la adopción de clones diferentes en cada sitio es necesario conocer la capacidad de los materiales genéticos para tolerar o evitar los estreses predominantes en cada uno y conocer los mecanismos de aclimatación que poseen. No obstante, la utilización de clones como material de plantación no es excluyente de las fuentes seminales en aquellas especies donde existen ambas estrategias, sino que pueden convivir. La oferta de nuevos clones permite variar el genotipo utilizado en las plantaciones, favorecer el establecimiento de un sistema más diversificado y realizarlo combinando siempre genotipos selectos (con alto potencial productivo 0 adaptabilidad a determinados ambientes).

La alta variabilidad que presentan las Salicáceas en general, y los álamos en particular, resulta un insumo importante para argumentar las hipótesis de interacciones intraespecíficas benéficas. La mezcla de clones de álamos permite combinar la operatividad de utilizar un mismo tipo de material de plantación (i.e., estacas o guías) con la búsqueda de rodales más diversos, que no solo resulten más estables ante factores de estrés ambientales, sino que realicen un uso complementario de los recursos. Las plantaciones clonales mixtas pueden favorecer a la disminución de los impactos negativos que tienen las actividades productivas en el ambiente, distribuir espacialmente la demanda de nutrientes del suelo, aumentar la diversidad genética y mejorar los índices de rendimiento del rodal. Estas consideraciones son aplicables a las Salicáceas en particular, pero factibles de extrapolar a otros sistemas forestales donde la silvicultura clonal ha avanzado.

\section{REFERENCIAS}

Almeida, I., C. Rösch \& S. Saha. 2018. Comparison of Ecosystem Services from Mixed and Monospecific Forests in Southwest Germany: A Survey on Public Perception. Forests 9 (10): 627.

Alvarez, J. A. 2018. Estabilidad productiva y plasticidad fenotípica de Populus spp. en relación con el contenido de agua y sales en el suelo en el Delta del Paraná, Argentina. Universidad de Buenos Aires.

Alvarez, J.A., C. Graciano, F. Gortari \& J.E. Gyenge. 2015. Efecto de la disponibilidad hídrica (sequía y 
anegamiento) y la incidencia de roya sobre la productividad de distintos clones de álamos en el delta del Paraná. Proyecto de Investigación Aplicada (PIA 10034) p.35-37.

Ammer, C. 2019. Diversity and forest productivity in a changing climate. New Phytologist 221(1): 50-66.

Apshara, S.E. 2017. Comparative study on clonal and seedling progenies of selected cocoa (Theobroma cacao L.) genotypes. Indian Journal of Horticulture 74(2): 168. Ashton, M.S. \& M.J. Kelty. 2018. The practice of silviculture: applied forest ecology. 10th edición. M.S. Ashton \& M.J. Kelty, Eds. Wiley.

Bauhus, J., D.I. Forrester, B. Gardiner, H. Jactel, R. Vallejo \& H. Pretzsch. 2017. Ecological Stability of MixedSpecies Forests. En Mixed-Species Forests. Pretzsch H., D. Forrester, \& J. Bauhus (Eds.). Berlin. pp. 337-382.

Benavides, R., F. Valladares, C. Wirth, S. Müller \& M. Scherer-Lorenzen. 2018. Intraspecific trait variability of trees is related to canopy species richness in European forests. Perspectives in Plant Ecology, Evolution and Systematics 36: 24-32.

Benbrahim, M., A. Gavaland \& J. Gauvin. 2000. Growth and Yield of Mixed Polyclonal Stands of Populus in ShortRotation Coppice. Scandinavian Journal of Forest Research 15(6): 605-610.

Binkley, D., J.L. Stape, W.L. Bauerle \& M.G. Ryan. 2010. Explaining growth of individual trees: Light interception and efficiency of light use by Eucalyptus at four sites in Brazil. Forest Ecology and Management 259(9): 1704-1713.

Bonnin, S.M., J.A. Alvarez, A.P. Moretti \& C. Graciano. 2018. Uso de luz y agua de clones comerciales de álamo para la diversificación de las plantaciones del Delta del Paraná. En Reunión Argentina de Ecología. Asociación Argentina de Ecología, Ed. Mar del Plata, Argentina. pp. 585.

Borodowski, E.D. 2014. Salicáceas en el Delta del Paraná: situación actual y perspectivas. Cuarto Congreso Internacional de Salicáceas. La Plata, Argentina. pp.1-13. Boyden, S., D. Binkley \& J.L. Stape. 2008. Competition among eucalyptus trees depends on genetic variation and resource supply. Ecology 89(10): 2850-2859.

Carnus, J.M., J. Parrotta, E. Brockerhoff, M. Arbez, H. Jactel, A. Kremer, ... \& B. Walters. 2006. Planted forests and biodiversity. Journal of Forestry 104(2): 65-77.

Carpineti, L. 2005. Importancia de la Silvicultura Clonal. Revista de Información Sobre Investigación y Desarrollo Agropecuario 5: 147-150.

Castañeda, F. 2004. Tendencias y perspectivas para las iniciativas de criterios e indicadores para la ordenación forestal sostenible. En Recursos Naturales y Ambiente. CATIE, Ed. Turrialba, Costa Rica. Volúmen 42, pp. 51-59. Cerrillo, T. 2014. Selección de seis nuevos clones de sauce (Salix spp.) para el Delta del Paraná. IV Congreso Internacional de Salicáceas en Argentina - Jornadas de Salicáceas. La Plata, Argentina. pp. 12.

Cortizo, S.C. 2005a. Álamos en el Delta del Paraná. En: Mejores árboles para más forestadores: el programa de producción de material de propagación mejorado y el mejoramiento genético en el Proyecto Forestal de Desarrollo. Norverto, C.A., Ed. Buenos Aires. pp. 137-160. Cortizo, S.C. 2005b. Roya del álamo en el Delta del Paraná. IDIA XXI Forestales 5(8): 139-142.

Cortizo, S., S. Monteverde, E. Fernandez Tschieder, M. Refort, C. Taraborelli, G. Keil \& N. Abbiati. 2014. Características técnicas de un nuevo genotipo de álamo para su inscripción en el Registro Nacional de Variedades. Actas Jornadas de Salicáceas. La Plata, Argentina. pp. 110.

Cortizo, S., S. Monteverde \& N. Abbiati. 2017. Caracterización de la madera de cinco clones de Populus deltoides seleccionados dentro del programa de mejoramiento de INTA. II Congreso Latinoamericano de Estructuras de Madera y II Congreso IberoLatinoamericano de la Madera en la Construcción. Junín, Buenos Aires, Argentina.

Edwards, D.M., M. Jay, F.S. Jensen, B. Lucas, M. Marzano, C. Montagne, ... \& G. Weiss. 2012. Public Preferences Across Europe for Different Forest Stand Types as Sites for Recreation. Ecology and Society 17(1): 27.

Elferjani, R., A. DesRochers \& F. Tremblay. 2014. Effects of mixing clones on hybrid poplar productivity, photosynthesis and root development in northeastern Canadian plantations. Forest Ecology and Management 327: 157-166.

Elferjani, R., A. DesRochers \& F. Tremblay. 2016. Plasticity of bud phenology and photosynthetic capacity in hybrid poplar plantations along a latitudinal gradient in northeastern Canada. Environmental and Experimental Botany 125: 67-76.

Faustino, L.I. 2013. Cambios en la arquitectura y fisiología de Pinus taeda en respuesta a la fertilización y al estrés por sequía. Tesis. Facultad Ciencias Agrarias y Forestales, Universidad Nacional de La Plata, La Plata, Argentina. 240 pp.

Faustino, L.I., N.M.L. Bulfe, M.A. Pinazo, S.E. Monteoliva \& C. Graciano. 2013. Dry weight partitioning and hydraulic traits in young Pinus taeda trees fertilized with nitrogen and phosphorus in a subtropical area. Tree Physiology 33(3): 241-251.

Faustino, L.I.; M.E., Rodriguez; J.A. Alvarez; G.N. Doffo; S.C. Cortizo \& C. Graciano. 2016. Rendimiento y extracción de nutrientes en estaqueros de álamo del Delta del Paraná fertilizados con nitrógeno o fósforo. Revista de la Facultad de Agronomía 115 (1): 179-190.

Felton, A., U. Nilsson, J. Sonesson, A.M. Felton, J.M. Roberge, T. Ranius, ... \& K. Wallertz. 2016. Replacing monocultures with mixed-species stands: Ecosystem service implications of two production forest alternatives in Sweden. Ambio 45: 124-139.

Fernández, M.E. \& J. Gyenge. 2009. Testing Binkley's hypothesis about the interaction of individual tree water use efficiency and growth efficiency with dominance patterns in open and close canopy stands. Forest Ecology and Management 257(8): 1859-1865.

Filyushkina, A., F. Agimass, T. Lundhede, N. Strange \& J.B. Jacobsen. 2017. Preferences for variation in forest characteristics: Does diversity between stands matter? Ecological Economics 140: 22-29.

Forrester, D. I. \& J. Bauhus. 2016. A Review of Processes Behind Diversity-Productivity Relationships in Forests. Current Forestry Reports 2(1): 45-61.

Foster, G.S., R.J. Rousseau \& W.L. Nance. 1998. Eastern cottonwood clonal mixing study: intergenotypic competition effects. Forest Ecology and Management 112: 9-22.

Gauchat, M.E., E. Belaber, E. Cappa, C. Vera Bravo, J.A. López, A. Aparicio, ... \& S.N. Marcucci Poltri. 2015. Subprograma Pinus y Pseudotsuga. En Domesticación y mejoramiento de especies forestales. Ed. INTA. pp. 21-45. 
Griffin, A. 2014. Clones or improved seedlings of Eucalyptus? Not a simple choice. International Forestry Review 16(2): 216-224.

Grossiord, C. 2019. Having the right neighbors: how tree species diversity modulates drought impacts on forests. New Phytologist 228: 142- 49.

Guarnaschelli, A.B., A.M. Garau, S.C. Cortizo, J.A. Alvarez \& J.H. Lemcoff. 2011. Respuestas diferenciales a la sequía en clones de Populus deltoides cultivados en el Delta del Paraná. Tercer Congreso Internacional de Salicáceas. Trabajo Técnico. Argentina. 9 pp.

Guet, J., F. Fabbrini, R. Fichot, M. Sabatti, C. Bastien \& F. Brignolas. 2015. Genetic variation for leaf morphology, leaf structure and leaf carbon isotope discrimination in European populations of black poplar (Populus nigra L.). Tree Physiology 35(8): 850-863.

Gundersen, V.S. \& L.H. Frivold. 2008. Public preferences for forest structures: A review of quantitative surveys from Finland, Norway and Sweden. Urban Forestry and Urban Greening 7(4): 241-258.

Guo, X.Y., Z.Y. Huang, A.C. Xu \& X.S. Zhang. 2011. A comparison of physiological, morphological and growth responses of 13 hybrid poplar clones to flooding. Forestry 84(1): 1-12.

Hamanishi, E.T., G.L.H. Barchet, R. Dauwe, S.D. Mansfield \& M.M. Campbell. 2015. Poplar trees reconfigure the transcriptome and metabolome in response to drought in a genotype- and time-of-daydependent manner. BMC Genomics 16(1): 1-16.

Hector, A. \& M. Loreau. 2001. Partitioning selection and complementarity in biodiversity experiments. Nature 412(July): 72-76.

Hooper, D.U., F.I. Chapin, J.J. Ewell, A. Hector, P. Inchausti, P. Lavorel, ... \& D.A. Wardle. 2005. Effects of biodiversity on ecosystem functioning: a consensus of current knowledge. Ecological Monographs 75: 3-35.

Instituto Nacional de Semillas. 2020. Catálogo Nacional de Cultivares. Disponible en https://gestion.inase.gov.ar/consultaGestion/gestiones. Último acceso: abril 2020.

Jactel, H., E. Brockerhoff \& P. Duelli. 2005. A test of the biodiversity-stability theory : meta-analysis of tree species diversity effects on insect pest infestations, and reexamination of responsible factors. En Ecological Studies. M. Scherer-Lorenzen, C. Körner, \& E. D. Schulze (Eds.). Ed. Springer, Berlín. Vol. 176. pp. 235-262.

Joly, C.A. \& R.M.M. Crawford. 1982. Variation in tolerance and metabolic responses to flooding in some tropical trees. Journal of Experimental Botany 33(4): 799809.

Jones, R.E. 2001. Mechanisms for locating resources in space and time: Impacts on the abundance of insect herbivores. Austral Ecology 26(5): 518-524.

Kelty, M.J. 2006. The role of species mixtures in plantation forestry. Forest Ecology and Management 233(2-3): 195204.

Kumar, V. 2016. Clonal forestry: Benefits and limitation. Van Sangyan 3(1): 26-29.

Lambers, H., F.S. Chapin III \& T.L. Pons. 2008. Plant Physiological Ecology (2nd ed.). Ed. Springer, New York. 604 pp.

Larjavaara, M. 2008. A Review on Benefits and Disadvantages of Tree Diversity. The Open Forest Science Journal 1(1): 24-26.
Lopez, O.R. \& T.A. Kursar. 1999. Flood tolerance of four tropical tree species. Tree Physiology 19(14): 925-932. Luquez, V., F. Achinelli \& S. Cortizo. 2012. Evaluation of flooding tolerance in cuttings of Populus clones used for forestation at the Paraná River Delta, Argentina. Southern Forests: A Journal of Forest Science 74(1): 61-70.

McCracken, A.R. \& W.N. Dawson. 1997. Growing clonal mixtures of willow to reduce effect of Melampsora epitea var. epitea. Forest Pathology 27(5): 319-329.

McCracken, A.R. \& W.N. Dawson. 1998. Short rotation coppice willow in Northern Ireland since 1973: development of the use of mixtures in the control of foliar rust (Melampsora spp.). European Journal of Forest Pathology 28(4): 241-250.

Medeiros, A.R., H. Nogueira Paiva, H. Garcia Leite, S. Nolasco Oliveira Neto, D.G. Streck Vendrúscolo \& F.T. Silva. 2015. Análise silvicultural e econômica de plantios clonais e seminais de Tectona grandis L. f., em sistema Taungya. Revista Árvore 39(5): 893-903.

Meza, A.R., J. Rodriguez, K.C. Gatti \& E.E. Espinoza. 2015. Propagación de árboles de teca Tectona grandis $L$. f. por miniestacas. Temas Agrarios 20(2): 43-48.

Mina, M., M.O. Huber, D.I. Forrester, E. Thürig \& B. Rohner. 2018. Multiple factors modulate tree growth complementarity in Central European mixed forests. Journal of Ecology 106(3): 1106-1119.

Moghaddam, E.R. 2014. Growth, Development and Yield in Pure and Mixed Forest Stands. International Journal of Advanced Biological and Biomedical Research 2(10): 2725-2730.

Nichols, J.D., M. Bristow \& J.K. Vanclay. 2006. Mixedspecies plantations: Prospects and challenges. Forest Ecology and Management 233(2-3): 383-390.

Park, Y., J.M. Bonga \& H. Moon. 2016. Vegetative propagation of forest trees. National Institute of Forest Science. 668 pp.

Park, Y., J.M. Bonga \& T.J. Mullin. 1998. Clonal Forestry. En Forests genetic and tree breeding. A.K. Mandal \& G.L. Gibson (Eds.). Ed. CBS. New Delhi. 1st Edición, pp. 143167.

Payn, T., J.M. Carnus, P. Freer-Smith, M. Kimberley, W. Kollert, S. Liu, ... \& M.J. Wingfield. 2015. Changes in planted forests and future global implications. Forest Ecology and Management 352: 57-67.

Peng, Y. J., Y.M. Dong, B.K. Tu, Z.X. Zhou, B. Zheng, L.W. Luo, ... \& K.B. Du. 2013. Roots play a vital role in flood-tolerance of poplar demonstrated by reciprocal grafting. Flora 208(8-9): 479-487.

Prado, J.A. 2015. Plantaciones forestales: más allá de los árboles. Ed. Colegio de Ingenieros Forestales de Chile. Chile. 166 pp.

Resende, R.T., G.E. Marcatti, D.S. Pinto, E.K. Takahashi, C.D. Cruz \& M.D.V. Resende. 2016. Intragenotypic competition of Eucalyptus clones generated by environmental heterogeneity can optimize productivity in forest stands. Forest Ecology and Management 380: 5058.

Resende, R.T., A.A.V. Soares, D.I. Forrester, G.E. Marcatti, A.R. dos Santos, E.K. Takahashi, ... \& H.G. Leite. 2018. Environmental uniformity, site quality and tree competition interact to determine stand productivity of clonal Eucalyptus. Forest Ecology and Management 410: 76-83. 
Rezende, G.D.S.P., M.D.V. de Resende \& T.F. de Assis. 2014. Eucalyptus Breeding for Clonal Forestry. En Challenges and Opportunities for the World's Forests in the 21st Century. T. Fenning (Ed.). Ed. Springer pp. 393-424. Ribe, R.G. 1989. The aesthetics of forestry: What has empirical preference research taught us? Environmental Management 13(1): 55-74.

Rodriguez, M.E. 2014. Respuestas fisiológicas y bioquímicas a la inundación en álamo (Populus spp.). Tesis. Facultad Ciencias Naturales y Museo, Universidad Nacional de la La Plata, La Plata, Argentina. 170 pp.

Russo, G., I. Beritognolo, M. Sabatti, J.M. Climent, M. Lauteri \& P. De Angelis. 2018. Functional relationships between leaves and stem across canopy layers in two contrasting clones of Populus nigra L. Plant Physiology and Biochemistry 133: 22-28.
Vargas, M. (2015) Bioeconomía Región NEA. Ministerio de Ciencia, Tecnología e Innovación, Argentina. Disponible en: https://www.youtube.com/watch?v=DkKBNyj5asQ\&featur e=emb_title Último acceso: noviembre 2020

Wu, H.X. 2018. Benefits and risks of using clones in forestry - a review. Scandinavian Journal of Forest Research 34(5): 352-359.

Xavier, A. \& R.L. da Silva. 2010. Evolução da sivicultura clonal de Eucalyptus no Brasil. Agronomia Costarricense 34(1): 93-98.

Zobel, B. \& J. Talbert. 1984. Applied forest tree improvement. Ed. Wiley. New York. 505 pp. 